\title{
Nível de atividade física em mulheres com câncer de mama que realizaram treinamento combinado
}

\section{Level of physical activity in women with breast cancer who conducted combined training}

\author{
Rafael dos Santos Coelho ${ }^{*}$, Andréa Dias Reis ${ }^{2}$
}

ARTIGO ORIGINAL | ORIGINAL ARTICLE

\begin{abstract}
Introdução. A prevalência de pacientes com câncer de mama cresce alarmantemente e os efeitos colaterais do tratamento influenciam na capacidade funcional das pacientes. Objetivo. Avaliar a influência do treinamento combinado (TC) na atividade laboral, nível de atividade física e efeitos adversos. Métodos. Estudo piloto controlado com 28 pacientes em tratamento de câncer de mama (30 a 59 anos), sendo alocadas em Grupo Experimental (GE), onde realizaram TC (aeróbico+resistido e flexibilidade) em 12 semanas e o Grupo Controle (GC) não realizou TC. Resultados. As pacientes do GE apresentaram aumento no nível de atividade física $(p=0,0086)$, diferente do GC que não obteve diferença significativa $(p=0,1573)$. Os efeitos adversos apresentaram diferença significativa $(p=0,0056)$ entre os grupos após 12 semanas de TC, contrário da atividade laboral que não apresentou diferença significativa $(p=0,065)$. Conclusão. O TC foi eficaz para aumento do nível de atividade física em pacientes com neoplasias da mama.

Palavras-chave: neoplasias da mama, exercício, doença.
\end{abstract}

\begin{abstract}
Introduction. The prevalence of patients with breast cancer grows alarmingly and side effects of treatment influence the functional capacity of patients. Goal. To evaluate the influence of combined training (CT) on labor activity, level of physical activity and adverse effects. Methods. Pilot controlled study with 28 patients in patients undergoing breast cancer treatment (30 to 59 years), being allocated in Experimental Group (EG), where they performed the CT (aerobic+resistance and flexibility) in 12 weeks and the control group (CG) did not perform CT. Results. The EG patients showed an increase in the level of physical activity $(p=0.0086)$, different from the CG not had a significant difference $(p=0.1573)$. Adverse effects showed significant differences $(\mathrm{p}=0.0056)$ between the groups after 12 weeks of physical training, unlike the labor activity that showed no significant difference $(\mathrm{p}=0.065)$. Conclusion. The physical training was effective to increase the level of physical activity in patients with breast cancer.

Keywords: breast neoplasms, exercise, disease.
\end{abstract}

\footnotetext{
${ }^{1}$ Faculdade Pitágoras, São Luís, Maranhão, Brasil.

${ }^{2}$ Universidade Estadual Paulista, São Paulo, São Paulo, Brasil.

* Autor correspondente: rafaelcoelho97@outlook.com
} 


\section{INTRODUÇÃO}

O tratamento do câncer de mama gera efeitos adversos, como a dor moderada ou intensa, que acomete cerca de $30 \%$ a $90 \%$ dos pacientes. A mastectomia provoca: dor, fadiga e desconforto. A quimioterapia pode restringir ou comprometer as atividades laborais (Garcia \& Guirro, 2005; Sousa, et al., 2013; Maieski \& Sarquis, 2007). O nível de atividade física também pode ser reduzido em mulheres com câncer de mama (Battaglini et al., 2004).

O exercício físico com ênfase no treinamento combinado (aeróbio+resistido) diminui os efeitos adversos das neoplasias, pois diminui a fadiga e frequência cardíaca, mantém a capacidade cardiopulmonar e melhora da qualidade de vida (Adamsen, L. et al. 2003; Galvão \& Newton, 2005; Battaglini, et al. 2004; Schmidt, et al. 2013). Este trabalho é importante, devido à escassez de estudos não farmacológicos para melhoria de mulheres com câncer de mama. O objetivo do estudo é avaliar o nível de atividade física, atividade laboral e efeitos adversos em pacientes com câncer mamário que realizaram treinamento combinado (TC).

\section{MÉTODO}

Este ensaio clínico controlado (Nobre; Bernardo \& Jatene, 2003) foi desenvolvido no Hospital do Câncer Aldenora Bello, na cidade de São Luís, no estado do Maranhão, onde participaram do estudo mulheres com 30 a 59 anos, que estiveram em tratamento de câncer mamário (radioterapia e quimioterapia) ou acompanhamento clínico e assinaram o Termo de Consentimento Livre e Esclarecido. A pesquisa obteve aprovação do Comitê de Ética em Pesquisa da Universidade Federal do Maranhão, com número 20665713.2.0000.5087.

\section{Participantes}

A pesquisa teve como critérios de inclusão: mulheres na faixa etária entre 30 até 59 anos, realizar tratamento ou acompanhamento de câncer mamário. Exclusão: praticar treinamento físico, gravidez, amamentação, não ter condições de comunicação ou locomoção, distúrbios mental, faltar três vezes consecutivas, desistência ou suspensão por determinação médica. A amostra foi alocada em dois grupos: 1) Grupo Experimental (GE) realizou o TC e tratamento hospitalar; 2) Grupo Controle (GC) tendo apenas o tratamento hospitalar.

\section{Instrumentos e Procedimentos}

O grau de instrução, renda familiar foram constatados na anamnese. O tipo de câncer de mama e fase do tratamento foram verificados no registro hospitalar. O nível de atividade física foi verificado com o Questionário Internacional de Atividade Física, versão curta, validado no Brasil (Pardini et al, 2001) e classificado como: muito ativo, ativo, insuficientemente ativo e inativo (Souza et al. 2015). O vínculo empregatício e efeitos adversos foram verificados na anamnese.

Protocolo do treinamento combinado: $\mathrm{O}$ programa de TC consistiu em exercícios aeróbicos+resistidos (3 sessões por semana supervisionados) e flexibilidade ( 2 sessões sem supervisão), com duração de 12 semanas. As sessões de alongamento foram intercaladas com as sessões do treinamento aeróbico+resistido. Houve familiarização para cada exercício e as progressões das cargas foram a cada 4 semanas, sendo da $1^{\mathrm{a}}$ a $4^{\mathrm{a}}$ semana: 50 a $60 \%$ da Frequência Cardíaca de Treino (FCT), peso corporal ou $1 \mathrm{~kg}$ nos halteres e caneleiras, banda elástica (theraband) de intensidade moderada, da $5^{\mathrm{a}}$ a $8^{\mathrm{a}}$ semana: 70 a $80 \%$ da FCT, aumento de $1 \mathrm{~kg}$ e theraband forte e da $9^{\mathrm{a}}$ a $12^{\mathrm{a}}$ semana: 80 a $90 \%$ da FCT e manutenção do treinamento resistido (WEINECK, 2003).

Cada sessão de treinamento aeróbico+resistido teve duração de 60 minutos, seguindo a ordem: 30 minutos no cicloergômetro, flexão e extensão de quadril, desenvolvimento de ombros, agachamento com bola suíça, tríceps francês e remada curvada. A sessão de treinamento de flexibilidade teve duração aproximada de 15 minutos.

O treinamento aeróbico foi controlado por meio da FCT conforme Karvonen; Kentala e Mustala (1957). A FCT foi verificada por meio do frequencímetro (Polar FT2). O teste de capacidade cardiorrespiratória foi realizado por meio do protocolo de Rampa adaptado por Neil, et al (2013) em cicloergômetro (marca ERGO FIT, modelo ERGO 167-FITC CYCLE) com carga 
inicial 15 watts durante 5 ' de aquecimento e recuperação de 3' com a mesma carga, os estágios tiveram de 70 a 90 rotações por minuto e foram mensuradas pressão arterial com o aparelho de coluna de mercúrio convencional $(\mathrm{BD} \AA)$, frequência cardíaca (Polar FT2) e percepção subjetiva de esforço com a escala de Borg (Infor Fisic) a cada 15" para encerrar os estágios e no repouso.

O protocolo de treinamento resistido foi de 3 séries para cada exercício com 12 repetições e intervalo de $1^{\prime}$ entre séries e repetições, a velocidade de execução foi de 3" na fase concêntrica e excêntrica (Battaglini et al. 2006). Os exercícios foram alternados por seguimento para grandes grupos musculares e a carga foi estipulada no teste de repetições máximas (Guedes \& Guedes, 2006).

O treinamento de flexibilidade foi ativo, sem dor, onde cada exercício teve duração de 20 " em 3 séries (WEINECK, 2003). Os exercícios de flexibilidade foram quatro para membros superiores e seis para inferiores.

\section{Análise estatística}

Os dados foram apresentados por média, desvio-padrão, frequência absoluta e relativa. $\mathrm{O}$ teste de Kolmogorov-Smirnov verificou a normalidade dos dados. $\mathrm{O}$ teste $\mathrm{F}$ foi usado para idade, estatura e peso, que tiveram variâncias iguais entre grupos, logo foi utilizado o teste $\mathrm{T}$ de
Student não pareado. O teste de Mann-Whitney verificou o grau de instrução, renda familiar, tipo de câncer e estágio do tratamento e acompanhamento. O teste de qui-quadrado e Mc Nemar verificaram o vínculo empregatício e teste de Wilcoxon nível de atividade física e efeitos adversos. O software utilizado foi o Stata 10.0 com um $\bar{\alpha}=5 \%$.

\section{RESULTADOS}

Do Participaram do estudo 28 pacientes (GE: 14 e GC: 14 ), pois houve na presente pesquisa três perdas (GE: um diagnóstico de distúrbio mental, GC: uma paciente faleceu e outra não realizou avaliação final). A listagem hospitalar continha 300 pacientes, porém 269 não participaram (59 não atendiam aos critérios de inclusão, 10 recusaram participar e 200 por outros motivos "condição financeira, tempo disponível, localização da moradia e óbito").

Os grupos apresentaram homogeneidade na idade $(p=0,5380)$, grau de instrução $(\mathrm{p}=0,5572)$, renda familiar $(\mathrm{p}=0,1982)$, tipo de neoplasia $(p=0,4766)$, fase do tratamento $e$ acompanhamento $(\mathrm{p}=0,4252)$.

O TC proporcionou diferença entre os grupos para nível de atividade física $(\mathrm{p}=0,0037)$, porém não foram diferentes no vínculo empregatício $(p=0,065)$. Já os efeitos adversos foram diferentes no tempo basal $(p=0,0096)$ e após o TC $(p=0,0056)$ (Tabela 1$)$.

Tabela 1

Nível de atividade física, atividade laboral e efeitos adversos em pacientes com câncer de mama $(n=28)$

\begin{tabular}{|c|c|c|c|c|c|c|c|c|}
\hline \multirow{3}{*}{$\begin{array}{l}\text { Nível de Atividade } \\
\text { Muito Ativa }\end{array}$} & \multicolumn{2}{|r|}{ GE } & \multicolumn{4}{|c|}{ GC } & \multicolumn{2}{|c|}{ GE X GC } \\
\hline & Basal & 12semanas & $\mathrm{p}$ valor & Basal & 12semanas & pvalor & Basal & 12semanas \\
\hline & & $6(42,86 \%)$ & \multirow{5}{*}{$0,0086^{* *}$} & 0 & 0 & & & \\
\hline Ativa & $12(85,71 \%)$ & $8(57,14 \%)$ & & $14(100 \%)$ & $12(85,71 \%)$ & \multirow{3}{*}{0,1573} & \multirow{4}{*}{0,1495} & \multirow{3}{*}{$0,0037^{*}$} \\
\hline $\begin{array}{l}\text { Insuficientemente } \\
\text { Ativa }\end{array}$ & $2(14,29 \%)$ & 0 & & 0 & $2(14,29 \%)$ & & & \\
\hline Fisicamente Inativo & 0 & 0 & & 0 & 0 & & & \\
\hline Vínculo Empregatício & & & & & & & & \\
\hline $\begin{array}{l}\text { Não } \\
\text { Sim }\end{array}$ & $\begin{array}{c}10(71,43 \%) \\
4(28,57 \%)\end{array}$ & $\begin{array}{l}9(64,29 \%) \\
5(35,71 \%)\end{array}$ & 0,1655 & $\begin{array}{c}13(92,86 \%) \\
1(7,14 \%)\end{array}$ & $\begin{array}{c}13(92,86 \%) \\
1(7,14 \%)\end{array}$ & \multirow[t]{2}{*}{0,1000} & \multirow[t]{2}{*}{0,1390} & 0,065 \\
\hline Efeitos Adversos & & & & & & & & \\
\hline Nenhum & 0 & $1(7,14 \%)$ & \multirow{9}{*}{0,6349} & 0 & 0 & \multirow{9}{*}{0,5347} & \multirow{9}{*}{$0,0096^{*}$} & \multirow{9}{*}{$0,0056^{*}$} \\
\hline 1 & $2(14,29 \%)$ & $4(28,57 \%)$ & & $1(7,14 \%)$ & $1(7,14 \%)$ & & & \\
\hline 2 & $5(35,71 \%)$ & $4(28,57 \%)$ & & $2(14,29 \%)$ & $2(14,29 \%)$ & & & \\
\hline 3 & $5(35,71 \%)$ & $1(7,14 \%)$ & & $1(7,14 \%)$ & $1(7,14 \%)$ & & & \\
\hline 4 & $1(7,14 \%)$ & $2(14,29 \%)$ & & $3(21,43 \%)$ & $2(14,29 \%)$ & & & \\
\hline 5 & $1(7,14 \%)$ & $2(14,29 \%)$ & & $3(21,43 \%)$ & $3(21,43 \%)$ & & & \\
\hline 6 & 0 & 0 & & $2(14,29 \%)$ & $3(21,43 \%)$ & & & \\
\hline 7 & 0 & 0 & & $1(7,14 \%)$ & $1(7,14 \%)$ & & & \\
\hline$\geq 8$ & 0 & 0 & & $1(7,14 \%)$ & $1(7,14 \%)$ & & & \\
\hline
\end{tabular}

Nota. $n(\%)$ frequência absoluta (frequência relativa), ${ }^{*} \mathrm{p}<0,05,{ }^{* *} \mathrm{p}<0,01$. 


\section{DISCUSSÃO}

O nível de atividade física habitual foi aumentado após o TC em pacientes com neoplasias da mama, esse aumento pode ser decorrente a adaptação fisiológica, pois o treinamento proporciona melhor capacidade cardiorrespiratória, eficiência no recrutamento neural, controle na estrutura articular e nos tecidos moles requisitados no movimento (Mcardle; Katch \& Katch, 2011; Amaral, et al, 2005).

A quantidade de efeitos adversos não houve diferença para o período de 12 semanas, no entanto esses efeitos adversos, como: dor, fadiga, depressão, vertigem, dentre outros, quando amenizados ou não se elevam no paciente já se obtém um bom resultado clinicamente, pois os fármacos do tratamento do câncer de mama geram diversos efeitos colaterais ao paciente (Leal; Cubero \& Auro, 2010).

$\mathrm{O}$ vínculo empregatício também não teve diferença após 12 semanas, o que torna preocupante, pois há gasto com os fármacos para alívio dos efeitos adversos e também o custo com o próprio tratamento do câncer, onde cerca de cinquenta e um mil reais são gastos por um paciente que realiza biópsia, setorectomia, radioterapia, quimioterapia e uso de tamoxifeno durante cinco anos (Kaliks et al., 2013).

Tanto o custo elevado do tratamento com o câncer de mama, quanto o aumento da prevalência e incidência de novos casos de câncer de mama demonstra uma preocupação na saúde pública. $\mathrm{O}$ modelo de medicina curativa tem perdurado, sendo pouco utilizada a medicina preventiva como abordagem para tratamento nas redes hospitalares. O treinamento físico pode ser um tratamento para o câncer de mama, priorizando uma melhoria de saúde em longo prazo (Instituto Nacional de Câncer, 2015 \& Luz, 1991).

As limitações deste estudo incluem o tamanho amostral, no entanto houve diferença significativa no nível de atividade física. O ponto forte do presente estudo foi a utilização do TC com exercício aeróbico, resistido e de flexibilidade em doze semanas, diferenciando dos demais estudos, pois não existi consenso na literatura sobre a prescrição ideal para pacientes com câncer de mama (Battaglini, et al, 2014).

\section{CONCLUSÕES}

O TC com exercícios aeróbicos, resistidos e de flexibilidade foi eficaz para o aumento do nível de atividade física em pacientes com neoplasias da mama. O TC pode ser utilizado como um tratamento adjuvante hospitalar para aumento do nível de atividade física, sendo uma proposta de tratamento preventivo e não somente imediatista na saúde pública.

\footnotetext{
Agradecimentos:

Fundação de Amparo à Pesquisa e ao Desenvolvimento Científico e Tecnológico do Maranhão (FAPEMA) e Coordenação de Aperfeiçoamento de Pessoal de Nível Superior (CAPES) pela bolsa de mestrado.
}

Conflito de Interesses:

Nada a declarar.

\begin{tabular}{l}
\hline Financiamento: \\
Nada a declarar \\
\hline
\end{tabular}

\section{REFERÊNCIAS}

Amaral, M. T. P. do et al. (2005). Orientação domiciliar: proposta de reabilitação física para mulheres submetidas à cirurgia por câncer de mama. Revista Ciências Médicas. 14(5), 405-413.

Adamsen, L. et al. (2003). Feasibility, physical capacity, and health benefits of a multidimensional exercise program for cancer patients undergoing chemotherapy. Support Care Cancer. 11, 707-716.

Battaglini, C. L et al. (2006). Efeitos do treinamento de resistência na força muscular e níveis de fadiga em pacientes com câncer de mama. Revista Brasileira de Medicina do Esporte. 12(3), 153-155.

Battaglini, C. L. et al. (2014). Twenty-five years of research on the effects of exercise training in breast cancer survivors: a systematic review of the literature. World Journal Clinic. Oncology. 10, 9-12.

Battaglini, C. L.; Botarro, M.; Campbell J. S. \& Simão, R. (2004). Atividade física e níveis de fadiga em pacientes portadores de câncer. Revista Brasileira de Medicina do Esporte. 10(2), 98-104.

Galvão, D. A.; \& Newton, R, U. Review of Exercise Intervention Studies in Cancer Patients. Journal Of Clinical Oncology. 23(4), 899-909.

Garcia, L. B.; \& Guirro, E. C. O. (2005) Efeitos da estimulação de alta voltagem no linfedema pós- 
mastectomia. Revista Brasileira de Fisioterapia. 9(2), 243-248.

Guedes, D. P. E.; Guedes, J. E. R. P. (2006) Manual prático para avaliação em educação física. ed. 1 . São Paulo: Manole.

Kaliks, R. A. (2013) Pacientes com câncer de mama oriundas do Sistema Único de Saúde tratadas no setor privado: custos de um piloto de parceria público-privada em oncologia. Einstein. 11(2), 216-223.

Karvonen, M. J.; Kentala, E.; \& Mustala, O. (1957) The effects of training on heart rate; a longitudinal study. Annales Medicinae Experimentalis et Biologiae Fenniae, Helsinki, 35(3), 307-315.

Leal, J. H. S.; Cubero, D. G.; \& Auro, D. G. (2010) Hormonioterapia paliativa em câncer de mama: aspectos práticos e revisão da literatura. Revista Brasileira Clínica Médica. 8(4), 338-343. São Paulo.

Luz, M. T. (1991) Notas sobre as políticas de saúde no Brasil de "transição democrática" -anos 80. Revista de Saúde Coletiva. 1(1).

Mcardle, W. D. ; Katch, F. I. ; \& Katch, V. L. (2011). Fisiologia do exercício: energia, nutrição e desempenho humano. Rio de Janeiro: Guanabara Koogan, ed. 7.

Maieski, V. M.; \& Sarquis, L. M. M. (2007) Mulheres com câncer de mama em quimioterapia e sua influência sobre o trabalho. Cogitare Enferm. 12(3), 346-352.

Neil, S. E.; Klika, R. J.; J.; Mickenzie, D. C.; \& Campbell, K. L. (2013). Cardiorespiratory and neuromuscular deconditioning in fatigued and non-fatigued breast cancer survivors, Support Care Cancer. 21, 873-881.

Nobre, M. R. C.; Bernardo, W. M.; \& Jatene, F. B. (2003) A prática clínica baseada em evidências. Parte I - questões clínicas bem construídas. Revista Associação Médica Brasileira. 49(4), 445449.

Pardini, R. (2001). Validação do Questionário Internacional de Nível de Atividade Física (IPAQ Versão 6): estudo piloto em adultos jovens brasileiros. Revista Brasileira Ciência e Movimento. 9, 45-51.

Schmidt, M. E. et al. (2013). Progressive resistance versus relaxation training for breast cancer patients during adjuvant chemotherapy: Design and rationale of a randomized controlled trial (BEATE study). Contemporary Clinical Trials. 34, 117-125.

Sousa, E. et al. (2013) Funcionalidade de Membro Superior em Mulheres Submetidas ao Tratamento do Câncer de Mama. Revista Brasileira de Cancerologia, 59(3) 409-417.B

Souza, I. E. C. D., Bonfante, I. L. P., Moraes Júnior, F. B. de, \& Lopes, W. A. (2015) Níveis de atividade física e estágios de mudança de comportamento de universitários da área de saúde. Revista Brasileira de Atividade Física e Saúde. 20(6), 608610.

Weineck, J. (2003) BTreinamento ideal. ed.9. São Paulo: Manole, 2003.

Todo o conteúdo da revista Motricidade está licenciado sob a Creative Commons, exceto quando especificado em contrário e nos conteúdos retirados de outras fontes bibliográficas. 